\title{
Distinct Temperature Dependence of Cholesteric Pitch in Lyotropic Cholesteric Solutions of Polypeptide
}

\author{
Hiroshi Kosho, Yasuyuki TanaKa, Toshihiro IChizUKa, Susumu KawaUchi, \\ and Junji WaTANABE
}

Department of Polymer Chemistry, Tokyo Institute of Technology,

Ookayama, Meguro-ku, Tokyo 152-8552, Japan

(Received June 30, 1998)

\section{KEY WORDS Polypeptide / Lyotropic Liquid Crystal / Cholesteric Helix / Temperature Dependence of} Pitch / Helix Sense Inversion /

Examination was made for the thermotropic liquid crystalline nature of $\alpha$-helical polypeptides. ${ }^{1-8}$ The type of liquid crystal formed was cholesteric because of the chirality of constituent macromolecules and several cholesteric properties were reported. One most interesting property is the strong temperature dependence of cholesteric pitches, and detailed studies were performed using the representative materials, poly [ $(\gamma$-benzyl L-glutamate)-co-( $\gamma$-dodecyl L-glutamate)s] (PBDoLG) which formed a cholesteric mesophase in a temperature region above $60-100^{\circ} \mathrm{C}^{5,7,8}$ For these materials, cholesteric pitch $(P)$ was comparable to the wavelength of visible light in an initial mesophase temperature region, increased substantially with rising temperature, and diverged at $T_{\mathrm{N}}$. As temperature was raised further, the cholesteric helical structure again appeared and the pitch decreased. The cholesteric sense inversion from righthanded helix to left-handed one was clarified to be responsible for this rewinding phenomenon. The temperature dependence of pitches is described by the following empirical equation

$$
1 / P=\left(1 / P_{0}\right)\left(T_{\mathrm{N}}-T\right) /\left(T-T_{\mathrm{C}}\right)
$$

where $1 / P_{0}$ and $T_{\mathrm{C}}$ are constants characteristic to the material. ${ }^{7,8}$

Equation 1 is similar to that deduced theoretically by Kimura et al. ${ }^{9,10}$ Although the cholesteric twist results directly from the asymmetric carbons, namely the dispersion force as presented by Goossens, ${ }^{11}$ they consider that in the specific polymeric system, the twist would be also forced by steric interaction due to the screw-like van der Waals surface. ${ }^{12}$ Both independently affect not only the magnitude but also the sense of cholesteric helix. In such a case, the temperature dependence of the pitch appears complicated and as one possibility, the thermally-induced sense inversion is expected if twist arises with opposite sense. The polypeptides may be a typical example of this class of materials since side chains surrounding the $\alpha$-helical main chain take the helical arrangement.

In this study, examination on the temperature dependence of cholesteric pitches was extended to lyotropic cholesteric mesophases of PBDoLG dissolved in dodecyl alcohol. Temperature dependence was similarly observed as in the thermotropic system and found to be well described by the eq 1 . Constants included in the eq 1 were uniquely determined for all cholesteric solutions with polymer concentrations from $100 \mathrm{vol} \%$ to $40 \mathrm{vol} \%$ and concentration dependence is shown.

\section{EXPERIMENTAL}

The polymer used here is PBDoLG with dodecyl content of $39 \%$, prepared by ester interchange reaction between poly( $\gamma$-benzyl L-glutamate) $(D P=250)$ and dodecyl alcohol described elsewhere. ${ }^{3}$ The dodecyl alcohol was used as supplied by Tokyo Kasei Kogyo Co., Ltd. The polymer was dissolved into dodecyl alcohol at temperatures above $60^{\circ} \mathrm{C}$. The volume fractions (vol\%) of polymers were evaluated from weight fractions (wt\%) assuming the additivity of densities of the two components; $1.17 \mathrm{~g} \mathrm{ml}^{-1}$ for PBDoLG and $0.83 \mathrm{~g} \mathrm{ml}^{-1}$ for dodecyl alcohol.

Cholesteric pitch was determined by circular dichroism (CD) using a JASCO automatic recording spectrometer $(\mathrm{J}-20)$ and microscopic observation of the finger-printed patterns with an Olympus BH-2 polarizing microscope. Helical sense of cholesterics was clarified from the sign of the $\mathrm{CD}$ and optical rotational dispersion curves by using $\mathrm{J}-20$. The details of these methods are described in previous papers. ${ }^{5,6}$

\section{RESULTS AND DISCUSSION}

We first examined phase behavior in the PBDoLGdodecyl alcohol system. PBDoLG with dodecyl content of $39 \%$ forms the hexagonal columnar phase in which polymers are packed into a hexagonal lattice and hexagonal columnar phase transforms to the cholesteric liquid crystal at $102^{\circ} \mathrm{C} .^{3,4,7}$ This transformation temperature, $T_{1}$, could be easily determined from X-ray observation and also followed by a clear DSC peak. ${ }^{3,4}$ $T_{1}$ measured on heating decreased from $102^{\circ} \mathrm{C}$ to $62^{\circ} \mathrm{C}$ with decrease in polymer concentration from $100 \mathrm{vol} \%$ to $40 \mathrm{vol} \%$.

Above $T_{1}$, an isotropic phase is separated from a cholesteric phase by a characteristic chimney region where two phases coexist. The critical concentration, the A point, ${ }^{13}$ where the cholesteric phase starts to appear from the isotropic phase, is around $17 \mathrm{vol} \%$ and $\mathrm{B}$ point where the isotropic phase disappears completely, around $38 \mathrm{vol} \%$. Below $T_{\mathrm{l}}$, isotropic and hexagonal columnar 


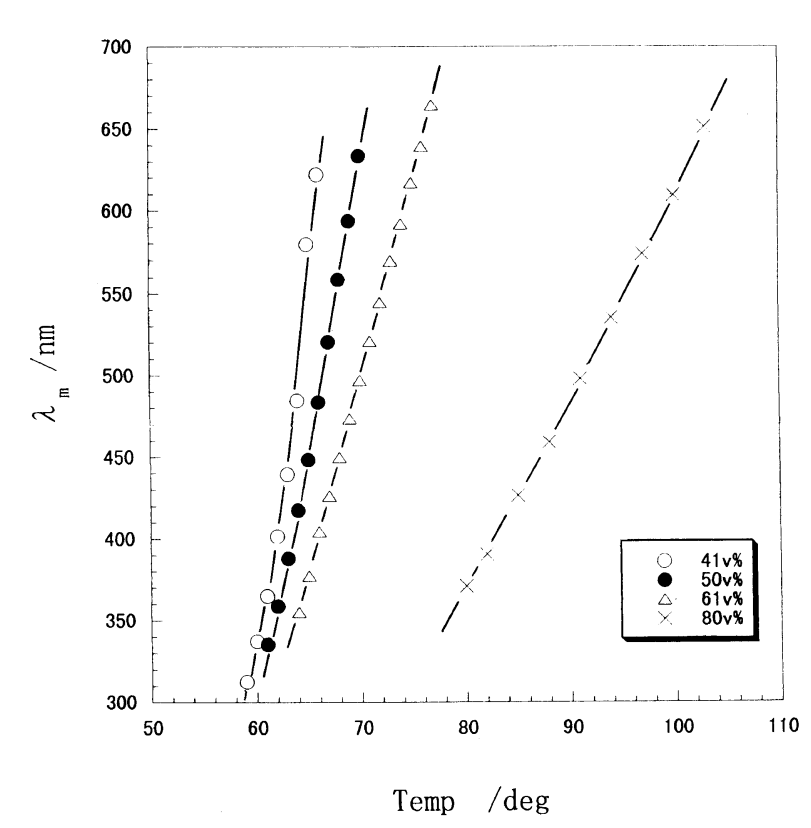

Figure 1. Temperature dependence of cholesteric optical pitch observed from circular dichroic spectra.

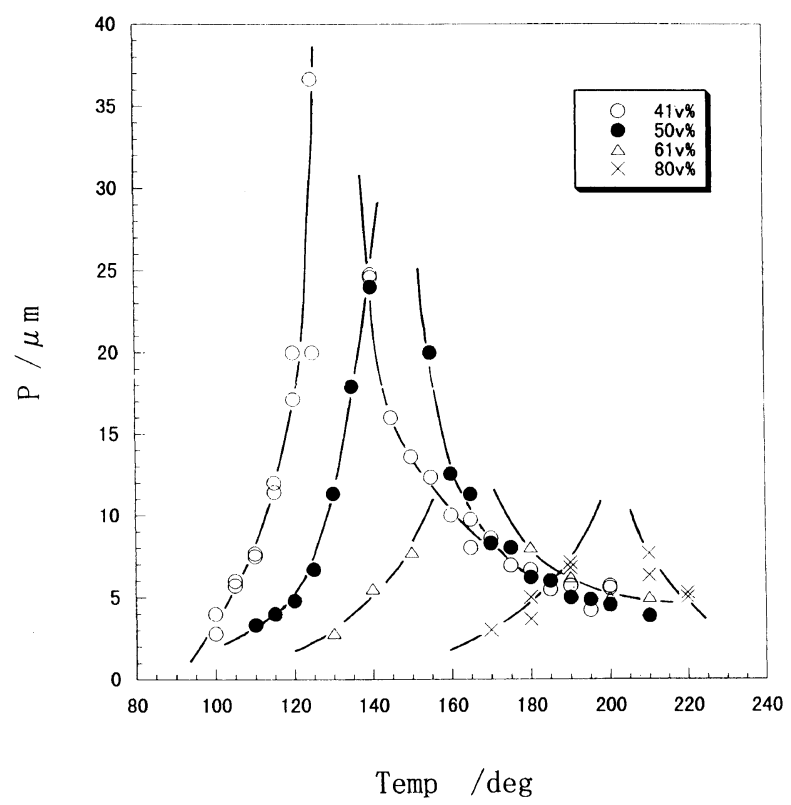

Figure 2. Temperature dependence of cholesteric pitch observed by the optical microscopy.

phases are observed. In the hexagonal columnar phase, polymer molecules with the solvents uniformly dispersed are packed into the two-dimensional hexagonal lattice above $\sim 50$ vol\%. ${ }^{14,15}$ Below 50 vol $\%$, the hexagonal phase of $\sim 50 \mathrm{vol} \%$ coexists with the isotropic phase. Thus, the cholesteric phases can be attained in the temperature range above $T_{\mathrm{I}}$ and in the concentration range above $38 \mathrm{vol} \%$. Detailed examination of phase behavior including the hexagonal columnar phase will be described elsewhere. ${ }^{16}$

Cholesteric solutions were prepared at polymer concentrations of $100,80,61,50$, and $41 \mathrm{vol} \%$. Relatively smaller pitches less than $700 \mathrm{~nm}$ were determined from the $C D$ reflection spectra. For this measurement, the cholesteric solution around $50 \mu \mathrm{m}$ was placed between glass plates and light was irradiated perpendicular to the film surface. The spectra had a negative sign and were very sharp, indicating that the right-handed macrohelix is well formed with the helical axes lying perpendicular to the glass surface. The wavelength at maximum peak of $\mathrm{CD}, \lambda_{\mathrm{m}}$, is the optical pitch and is related to $P$ by the equation

$$
\lambda_{\mathrm{m}}=n P
$$

where $n$, the refractive indices, are $1.51,1.50,1.49,1.48$, and 1.47 for solutions of $100,80,61,50$, and $41 \mathrm{vol} \%$, respectively. Figure 1 shows variation of $\lambda_{\mathrm{m}}$ with temperature. The cholesteric phase with $P$ comparable to the wavelength of visible lights appears in the lower temperature region as the polymer concentration decreases. Temperature variation of $P$ is more remarkable for lower concentrated solutions. Pitches change through the visible wavelength region from $300 \mathrm{~nm}$ to $700 \mathrm{~nm}$ within a temperature span of $4-5$ degrees for solutions with the lowest concentration of $41 \%$.

Figure 2 shows the temperature dependence of $P$ detected by optical microscopy. The pitch increased with temperature and diverged to result in unwinding of the cholesteric helix at the characteristic temperature of $T_{\mathrm{N}}$. On further heating, the helical structure with lefthanded helical sense reappeared and the helical pitch decreased. All solutions showed similar trend although $T_{\mathrm{N}}$ decreased substantially with decrease of the polymer concentration.

Figure 3 shows the overall temperature dependence of reciprocal cholesteric pitches, $1 / P$, proportional to the twist angle between adjacent pseudo-nematic layers. The data points given by the filled circles were obtained from the CD spectra while the open circles were determined by microscopic observation. Positive and negative values correspond to the right-handed and left-handed cholesteric helices, respectively, and zero at $T_{\mathrm{N}}$ indicates compensation for twist. All solutions showed similar temperature dependence of the reciprocal pitches. Obviously, the temperature dependence of the reciprocal pitches can be approximated by the hyperbolic curve of the eq 1 where the reciprocal pitch diverges at $T=T_{\mathrm{C}}$ on cooling while it approaches $-1 / P_{0}$ on heating after the sense inversion of cholesteric helix at $T_{\mathrm{N}}$. In practice, the data points fell on the solid curve obtained by curve fitting with the eq 1 (see Figure 3). The correspondence was fairly good, and $T_{\mathrm{N}}, T_{\mathrm{C}}$ and $1 / P_{0}$ uniquely determined are listed in Table $\mathrm{I}$ and plotted against polymer concentration in Figure 4. Systematic variation of $T_{\mathrm{N}}$, $T_{\mathrm{C}}$, and $1 / P_{0}$ with polymer concentration was observed. $T_{\mathrm{C}}$ and $1 / P_{0}$ decreasd and $T_{\mathrm{N}}$ increased with the polymer concentration.

Subsequent to the studies by Uematsu and Uematsu, ${ }^{17}$ the temperature dependence of pitch in the polypeptide lyotropic system has been shown to follow the empirical equation,

$$
1 / P=a\left(1-T / T_{\mathrm{N}}\right)
$$

The present data are thus exceptional. Comparing eq 1 with eq 3 , one notices that eq 1 can be rewritten in the same form as eq 3 in the limited temperature region of $T \approx T_{\mathrm{N}}$. Then, $a$ is given by $\left(1 / P_{0}\right) /\left(1-T_{\mathrm{C}} / T_{\mathrm{N}}\right)$. Values of $a$ calculated are listed in the last column of Table I. The 

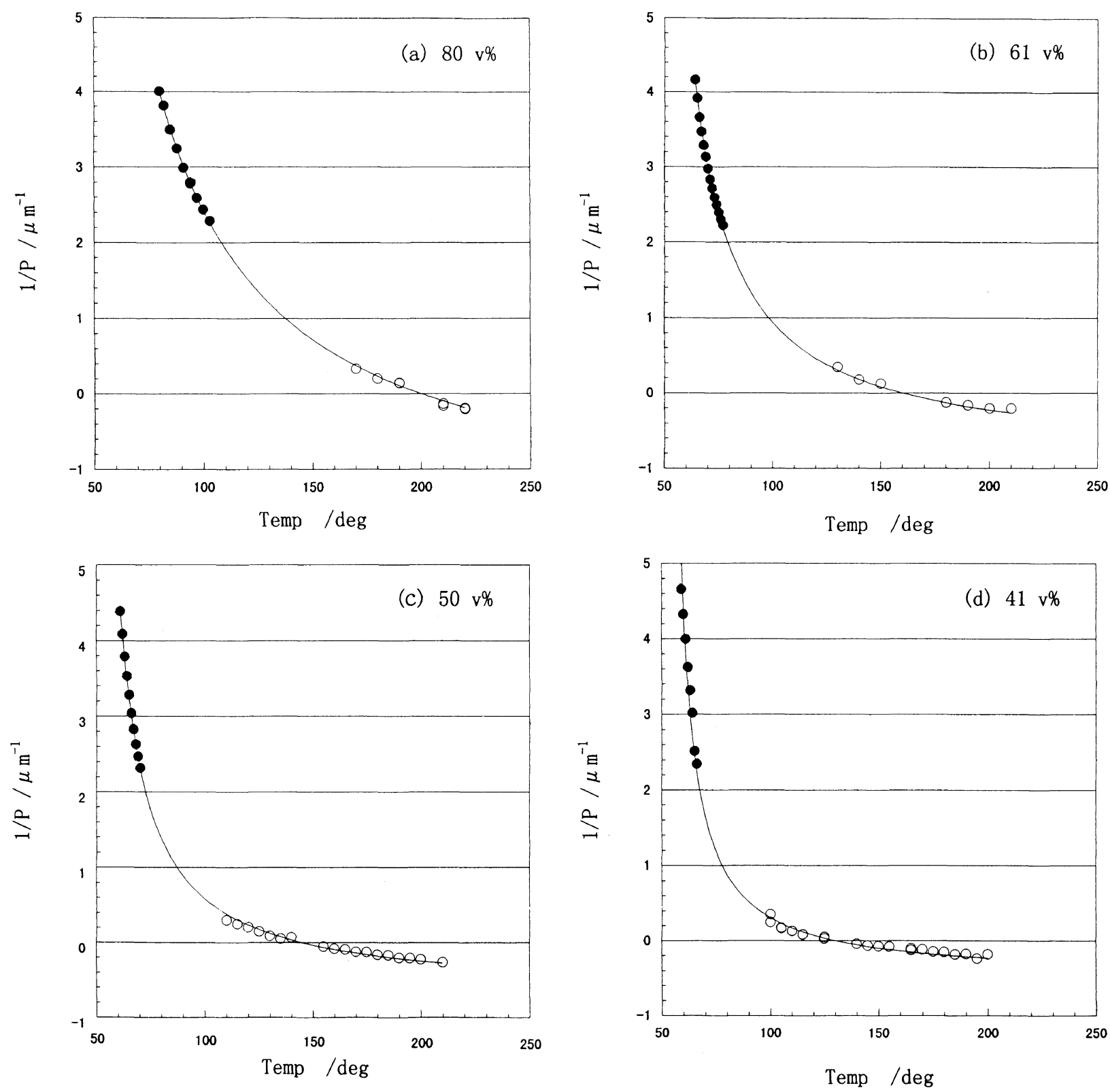

Figure 3. Temperature dependence of reciprocal pitch. Data points as open and filled circles were obtained by optical microscopic and circular dichroic methods, respectively. Positive and negative signs indicate right- and left-handed helices, respectively. Solid curves were obtained using eq 1.

Table I. Parameters, $1 / P_{0}, T_{\mathrm{N}}, T_{\mathrm{C}}$, and $a$ in eq 1 and 3 as determined from curve fitting with observed data

\begin{tabular}{ccccc}
\hline Polymer concentration/vol\% & $1 / P_{\mathrm{O}} / \mathrm{nm}^{-1}$ & $T_{\mathrm{N}} / \mathrm{K}$ & $T_{\mathrm{C}} / \mathrm{K}$ & $a^{\mathrm{a}} / \mathrm{nm}^{-1}$ \\
\hline 100 & $3.6 \times 10^{-3}$ & 498 & 302 & $9.1 \times 10^{-3}$ \\
80 & $1.8 \times 10^{-3}$ & 473 & 300 & $4.9 \times 10^{-3}$ \\
61 & $8.8 \times 10^{-4}$ & 433 & 317 & $3.3 \times 10^{-3}$ \\
50 & $6.7 \times 10^{-4}$ & 418 & 321 & $2.9 \times 10^{-3}$ \\
41 & $4.9 \times 10^{-4}$ & 403 & 325 & $2.5 \times 10^{-3}$
\end{tabular}

${ }^{a}$ Corresponding to $\left(1 / P_{0}\right) /\left(1-T_{\mathrm{C}} / T_{\mathrm{N}}\right)$.

approximate values are obtained for lyotropic systems. ${ }^{17}$ We first speculated that temperature dependence expressed by eq 1 is characteristic of thermotropic polypeptides, but there is no reason to distinguish the temperature dependence of the pitch of a lyotropic system from that of a thermotropic system. Possibly, cholestetic pitch in the lyotropic systems has been so far measured

Polym. J., Vol. 31, No. 2, 1999

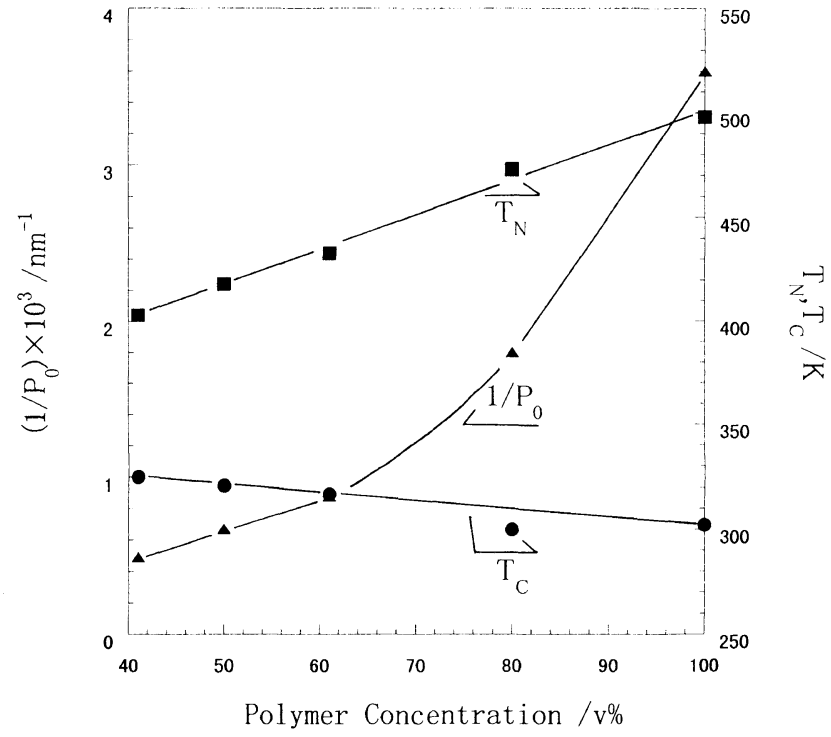

Figure 4. Plots of $1 / P_{0}, T_{\mathrm{N}}$, and $T_{\mathrm{C}}$ against polymer concentration 


\section{H. KosHo et al.}

only in the limited temperature region near $T_{\mathrm{N}}$ and the eq 1 would be applicable to any lyotropic systems if observation is done over a wide temperature region. More detailed studies on this point are now in progress.

\section{REFERENCES}

1. J. Watanabe, Y. Fukuda, R. Gehani, and I. Uematsu, Macromolecules, 17, 1004 (1984).

2. J. Watanabe, H. Ono, I. Uematsu, and A. Abe, Macromoecules, 18, 2141 (1985).

3. J. Watanabe, M. Goto, and T. Nagase, Macromolecules, 20, 298 (1987).

4. J. Watanabe and T. Nagase, Polym. J., 19, 781 (1987)

5. J. Watanabe and T. Nagase, Macromolecules, 21, 171 (1988).

6. J. Watanabe, T. Nagase, H. Itoh, T. Ishii, and T. Satoh, Mol. Cryst. Liq. Cryst., 164, 135 (1988)
7. J. Watanabe, T. Nagase, and T. Ichizuka, Polym. J., 22, 1029 (1990).

8. J. Watanabe, "Ordering in Macromolecular Systems," A. Teramoto, M. Kobayashi, and T. Norisue, Ed., Springer, Berlin, 1993, pp 99-113.

9. H. Kimura, M. Hoshino, and H. Nakano, J. Phys (Les Vlis, Fr.), 40, C3-174 (1976).

10. H. Kimura, M. Hoshino, and H. Nakano, J. Phys. Soc. Jpn., 51 1584 (1982).

11. W. J. A. Goossens, Mol. Cryst. Liq. Cryst., 12, 237 (1971).

12. J. P. Straley, Phys. Rev., A, 14, 1835 (1976).

13. E. L. Wee and W. G. Miller, Nature, 346, 44 (1990)

14. J. Watanabe and Y. Takashina, Macromolecules, 24, 3423 (1991).

15. A. Takada, K. Fujii, J. Watanabe, T. Fukuda, and T. Miyamoto, Macromolecules, 27, 1651 (1994)

16. J. Watanabe, to be published.

17. I. Uematsu and Y. Uematsu, Adv. Polym. Sci., 59, 37 (1984). 